\title{
Low-energy electron impact dissociative recombination and vibrational transitions of $\mathrm{N}_{2}^{+}$
}

\author{
A. Abdoulanziz, ${ }^{1}$ C. Argentin, ${ }^{1,2}$ V. Laporta, ${ }^{1,3,4}$ K. Chakrabarti, ${ }^{5}$ A. Bultel, ${ }^{2}$ J. Tennyson, ${ }^{4,1}$ I. F. \\ Schneider, ${ }^{1,6, \text { a) }}$ and J. Zs. Mezei ${ }^{1,7, b)}$ \\ 1) LOMC-UMR6294 CNRS-Université Le Havre Normandie, 76058 Le Havre, France \\ ${ }^{2)}$ CORIA-UMR6614 CNRS-Université de Rouen Normandie, 76800 Saint-Etienne du Rouvray, \\ France \\ 3) P.Las.Mi Lab CNR-Nanotec, 70126 Bari, Italy \\ 4) Dept. of Physics and Astronomy, University College London, WC1E 6BT London, UK \\ ${ }^{5)}$ Dept. of Mathematics, Scottish Church College, 700006 Kolkata, India \\ 6) LAC-FRE2038, CNRS-Université Paris-Saclay, 91405 Orsay, France \\ 7) Institute for Nuclear Research, 4001 Debrecen, Hungary
}

(Dated: 20 November 2020)

Cross sections and thermal rate coefficients are computed for electron-impact dissociative recombination and vibrational excitation/de-excitation of the $\mathrm{N}_{2}^{+}$molecular ion in its lowest six vibrational levels, for collision energies/temperatures up to $2.3 \mathrm{eV} / 5000 \mathrm{~K}$.

\section{INTRODUCTION}

The nitrogen molecule $\mathrm{N}_{2}$ is one of the most widely studied species so far in plasma physics. Being very stable at low temperature, it is very abundant in the Earth atmosphere, and is notably present in other planetary atmospheres - Ti$\tan 98.4 \%{ }^{1}$, Triton ${ }^{2}$ Pluto $^{3}$, Venus $3.5 \%$ and Mars $1.9 \% 1$

. For other trans-Neptunian objects than Pluto, this molecule is also one of the main component of the ices - spectroscopically observed at their surfaces - and may produce a very thin atmosphere when the temperature increases under solar irradiation ${ }^{4}$. Under the influence of an electric field, the high altitude planetary atmosphere can be crossed by few milliseconds giant discharges called sprites, whose spectroscopic signature is mainly due to spontaneous emission from $\mathrm{N}_{2}$ excited electronic states ${ }^{5}$.

Consequently, the $\mathrm{N}_{2}^{+}$cation is also of huge interest. Due to the solar irradiation, the production of $\mathrm{N}_{2}^{+}$on excited vibrational states plays a significant role in the characteristics of the Earth's thermosphere ${ }^{6}$. It is also the main molecular cation in the atmosphere of $\operatorname{Titan}^{7}$ and Triton ${ }^{8}$. On the other hand, during the atmospheric entry of a spacecraft in Earth's and Titan's atmospheres, the hypersonic compression of the gases leads to the formation of a plasma departing from local thermodynamic equilibrium ${ }^{9}$. The ionic composition, including $\mathrm{N}_{2}^{+}$, plays a key role in the radiation emitted by the plasma in the near UV spectral region ${ }^{10}$. In many plasmaassisted industrial processes elaborated so far, the plasma reactivity is greatly enhanced by the presence of $\mathrm{N}_{2}^{+}$. This is, for instance, the case in the ammonia synthesis in plasmas/liquid processes $^{11}$. $\mathrm{N}_{2}^{+}$is also very effective in the antibacterial treatment of polyurethane surfaces ${ }^{12}$. Moreover, $\mathrm{N}_{2}^{+}$- as $\mathrm{N}_{2}$ - is a key ingredient in the steel nitriding, resulting in the improving of its frictional wear resistance, surface hardness and corrosion resistance ${ }^{13}$. Furthermore, $\mathrm{N}_{2}^{+}$also plays a major role

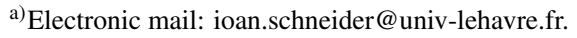

b)Electronic mail: mezei.zsolt@atomki.hu.
}

in the dermatological treatments based on the nitrogen radiofrequency discharges ${ }^{14}$.

The characteristics of the nitrogen-containing plasmas cannot be fully understood without a deep knowledge of the reactivity of $\mathrm{N}_{2}^{+}$, in particular by collisions with electrons.

Dissociative Recombination (DR) is the major molecular cation destruction reaction, that takes place when an electron collides with the $\mathrm{N}_{2}^{+}$molecular cation, leading to neutral atomic fragments:

$$
\mathrm{N}_{2}^{+}\left(v_{i}^{+}\right)+e^{-}(\varepsilon) \longrightarrow \mathrm{N}+\mathrm{N}
$$

Here $\varepsilon$ is kinetic energy of the incident electron and $v_{i}^{+}$the initial vibrational quantum number of the target.

In the same time other competitive processes might occur:

$$
\mathrm{N}_{2}^{+}\left(v_{i}^{+}\right)+e^{-}(\varepsilon) \longrightarrow \mathrm{N}_{2}^{+}\left(v_{f}^{+}\right)+e^{-}\left(\varepsilon_{f}\right),
$$

i.e. elastic (EC) $\left(v_{f}^{+}=v_{i}^{+}\right)$, inelastic (IC) $\left(v_{f}^{+}>v_{i}^{+}\right)$and superelastic (SEC) collisions $\left(v_{f}^{+}<v_{i}^{+}\right), v_{f}^{+}$standing for the final vibrational quantum number of the target ion. These processes are also known as Elastic Scattering (ES), Vibrational Excitation (VE) and Vibrational deExcitation (VdE) respectively.

The elementary non-thermal electron driven processes, in particular dissociative recombination, was experimentally studied using plasmas with laser induced photo-fluorescence techniques ${ }^{15}$, shock tubes ${ }^{16}$, discharge afterglow experiments $^{17,18}$ and microwave techniques ${ }^{19}$. The most detailed collisional data can be obtained in merged beam ${ }^{20}$ and/or storage ring experiments ${ }^{21}$.

Two different sets of theoretical calculations have been performed ${ }^{22-25}$ on the DR of $\mathrm{N}_{2}^{+}$. They involved different quantum chemistry and similar nuclear dynamics calculations, and both studies were focusing on the ground and the lowest three vibrational levels of the target.

Our aim with this paper is to extend as far as possible the calculations started in ${ }^{22}$. This extension refers to:

i) the kinetic energy of the incoming electron: up to $2.3 \mathrm{eV}$ vs $1 \mathrm{eV}$ previously. 
ii) the elementary processes explored: besides the DR studied in the past, the EC, VE and VdE cross sections and rate coefficients are computed.

iii) the vibrational levels considered in the vibrational transitions: up to the fifth excited level of the target $v s$ the third previously, and the lowest ten vibrational levels as final ones. The rotational effects have been neglected, since they are important only at very low collision energies.

All these extensions make our results relevant for the atmospheric and cold plasma environments, electron temperatures where the rotational effects can be neglected.

The paper is organized as follows: After a brief description of the theoretical approach (section II), we present in more details the molecular data used in the calculations (section III) followed by the presentation of the results (section IV). The paper is ended by conclusions.

\section{THEORETICAL APPROACH}

The efficiency of our method of modeling the electron/diatomic cation collisions, based on the Multichannel Quantum Defect Theory (MQDT) has been proved in many previous studies on different species, including $\mathrm{H}_{2}^{+}$and its isopologues ${ }^{26-28}, \mathrm{ArH}^{+29}, \mathrm{CH}^{+30}, \mathrm{SH}^{+31}$, etc. The general ideas of our approach were already presented in detail in our previous study of the $\mathrm{N}_{2}{ }^{+}$dissociative recombination ${ }^{22}$ and, therefore, here we restrict ourselves to its major steps.

The reactive processes (1) and (2) involve ionization channels - describing the scattering of an electron on the target cation - and dissociation channels - accounting for atom-atom scattering. The mixing of these channels results in quantum interference of the direct mechanism - in which the capture takes place into a doubly excited dissociative state of the neutral system - and the indirect one - in which the capture occurs via a Rydberg bound state of the molecule belonging to a closed channel, this state being predissociated by the dissociative one. In both mechanisms the autoionization - based on the existence of open ionization channels - is in competition with the predissociation, and can lead to the excitation or to the de-excitation of the cation.

One starts with the building of the interaction interaction matrix $\mathscr{V}$ that drives the collision, whose elements quantify the couplings between the different channels - ionization and/or dissociation ones.

More specifically, each of the ionization channels, built on the $\mathrm{N}_{2}^{+}$ion in one of its three lowest electronic states $-X^{2} \Sigma_{g}^{+}$, $A^{2} \Pi_{u}$ or $B^{2} \Sigma_{u}^{+}$, see Fig. 1 - and on its vibrational level, interacts with all the dissociation exit channels (Rydbergvalence interaction), but also with the other ionization channels (Rydberg-Rydberg interactions) - Fig. 2. Depending on the total energy of the system these ionization channels can be open - the entrance channels, describing the incident electron colliding the ion in its ground electronic state, or exit channels, describing the auto-ionization, i.e. elastic scattering, vibrational excitation and de-excitation - or closed - describing the resonant temporary captures into Rydberg states.

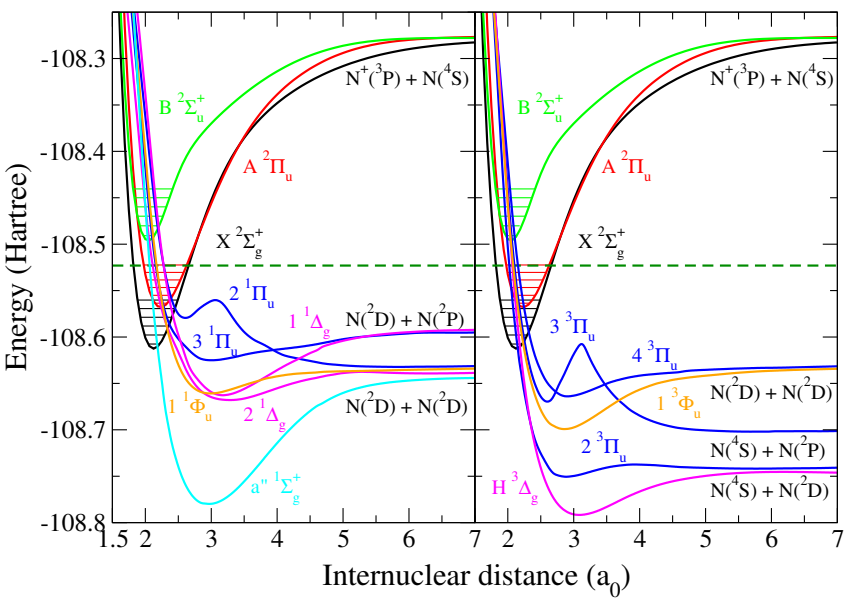

FIG. 1. Potential energy curves (PEC) relevant for the DR of $\mathrm{N}_{2}^{+22}$. Target cation $\mathrm{N}_{2}^{+}$: ground electronic state $\left(X^{2} \Sigma_{g}^{+}\right)$black, first excited state $\left(A^{2} \Pi_{u}\right)$ red, second excited state $B^{2} \Sigma_{u}^{+}$green. Neutral system $\mathrm{N}_{2}$ : Left panel, singlet states of different symmetries - blue for ${ }^{1} \Pi_{u}$, magenta for ${ }^{1} \Delta_{g}$, cyan for $a^{\prime 1} \Sigma_{g}^{+}$and orange for ${ }^{1} \Phi_{u}$. Right panel, triplet states of different symmetries - blue for ${ }^{3} \Pi_{u}$, magenta for $H^{3} \Delta_{g}$ and orange for ${ }^{3} \Phi_{u}$. The lowest five vibrational levels of each electronic state of the ion and the dissociative asymptotic limits for all states are shown. The green dashed line gives the upper limit of the total energy of the system, above which our results are still reasonably correct (see text).

Once the $\mathscr{V}$-matrix is elaborated, we build the short-range reaction matrix $\mathscr{K}$ of the collision, as a second order perturbative solution of the Lippmann-Schwinger equation. The diagonalized version of the $\mathscr{K}$-matrix (in the eigenchannel representation) whose eigenvalues are expressed in term of long range phase-shifts of the eigenfunctions, together with the vibronic couplings between the ionization channels, serve for the building of the frame transformation matrices.

Applying a Cayley transformation on these latter matrices we can set up the generalized scattering matrix $X$. The Seaton's method of 'eliminating' the closed channels ${ }^{32}$ is then employed, resulting in the physical scattering matrix $\mathscr{S}$ :

$$
S=X_{o o}-X_{o c} \frac{1}{X_{c c}-\exp (-\mathrm{i} 2 \pi v)} X_{c o},
$$

relying on the block-matrices involving open $\left(X_{o o}\right)$, open and closed $\left(X_{o c}\right.$ and $\left.X_{c o}\right)$ and closed $\left(X_{c c}\right)$ channels. The diagonal matrix $v$ in the denominator of equation (3) contains the effective quantum numbers corresponding to the the vibrational thresholds of the closed ionisation channels at given total energy of the system.

Finally, the cross section for the dissociative recombination and for the vibrational transitions - vibrational excitation/ deexcitation and elastic scattering write respectively as:

$$
\sigma_{\text {diss } \leftarrow v_{i}^{+}}=\frac{\pi}{4 \varepsilon} \rho^{s y m} \sum_{l, j}\left|S_{d_{j}, l v_{i}^{+}}^{\Lambda}\right|^{2}
$$




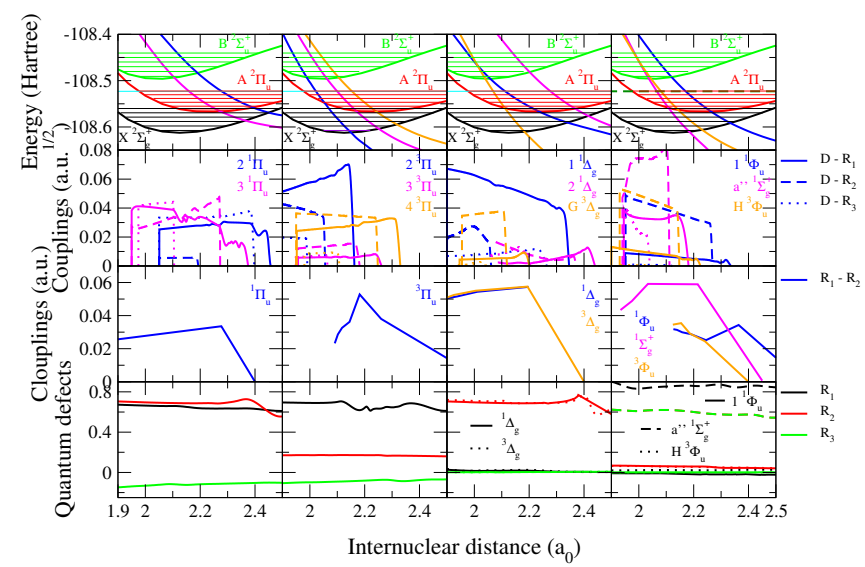

FIG. 2. Molecular data sets for the modeling of reactive collisions between electrons and $\mathrm{N}_{2}^{+22}$. $1^{\text {st }}$ row: PECs of the relevant states of the ion and of the neutral for all relevant symmetries. $2^{\text {nd }}$ row: Rydberg-valence electronic couplings. $3^{\text {rd }}$ row: Rydberg-Rydberg electronic couplings. $4^{\text {th }}$ row: Quantum defects characterizing the Rydberg series of states.

and

$$
\sigma_{v_{f}^{+} \leftarrow v_{i}^{+}}=\frac{\pi}{4 \varepsilon} \rho^{s y m} \sum_{l, l^{\prime}}\left|S_{l^{\prime} v_{f}^{+}, l v_{i}^{+}}^{\Lambda}-\delta_{l, l^{\prime}} \delta_{v_{i}^{+}, v_{f}^{+}}\right|^{2}
$$

where $d_{j}$ stands for a given dissociative state and $\rho^{s y m}$ the ratio between the state-multiplicities of the neutral and the target ion.

\section{MOLECULAR DATA}

The nuclear dynamics in low-energy electron/molecular cation collisions crucially depends on the molecular structure of the target and of the formed neutral - often superexcited - complex. The relevant molecular data sets consist in the potential energy curves (PECs) of the target cation - for the ground and for the excited electronic states - the PECs of the doubly excited bound or dissociative molecular states of the neutral, the quantum defect-functions characterizing the bound mono-excited Rydbers series of the neutral, and the coupling functions between the several - ionization and dissociation - continua.

One of the few quantum chemistry methods capable to produce the highly excited molecular states at the required accuracy is based on the R-Matrix Theory ${ }^{33}$. The bound and resonant adiabatic potential energy curves of the valence and Rydberg states of $\mathrm{N}_{2}$ having singlet and triplet symmetries have been published in Refs. ${ }^{34,35}$. The diabatic curves, couplings and quantum defects relevant for the dissociative recombination of $\mathrm{N}_{2}^{+}$were presented $\mathrm{in}^{22}$. The electronic states of the target were calculated using the standard quantum chemistry program suite Molpro ${ }^{36}$. Figure 1 shows the PECs of the dissociative molecular states of $\mathrm{N}_{2}$, as well as those of the rele-
TABLE I. The energies of the vibrational levels of the $\mathrm{N}_{2}^{+}$molecular cation - relative to the ground one - involved either as initial or as final levels in the present calculations.

\begin{tabular}{c|cccccccccc}
\hline \hline$v^{+}$ & 0 & 1 & 2 & 3 & 4 & 5 & 6 & 7 & 8 & 9 \\
\hline$E_{v^{+}}(\mathrm{eV})$ & 0.0 & 0.266 & 0.528 & 0.786 & 1.040 & 1.290 & 1.536 & 1.777 & 2.014 & 2.248 \\
\hline \hline
\end{tabular}

vant states of $\mathrm{N}_{2}^{+}$, involved in our previous ${ }^{22}$ and present calculations.

The same PECs are dispatched by symmetries in the first row of Figure 2, figure which contains the whole ensemble of molecular data relevant for the modeling of the internuclear dynamics. Whereas its first row illustrates how favorable are the crossings between the PECs of the dissociative states with those of the target ones - i.e. the Franck-Condon effect the driving interactions of the dynamics - the Rydberg-valence couplings - are shown in the second row. The third row gives the Rydberg-Rydberg couplings: In the present calculation, only the couplings among the series correlating to the ground $(X)$ and first excited $(A)$ state of the ion have been considered. And finally the last row of the figure displays the quantum defects characterizing the Rydberg series built, each of them, on one of the three cores X, A and B.

\section{RESULTS AND DISCUSSIONS}

Based on the molecular data already presented in fig. 2, we have performed the nuclear dynamics calculations using the MQDT approach presented in Section II. The DR, EC, VE and $\mathrm{VdE}$ cross sections have been calculated considering the $\mathrm{N}_{2}^{+}$target in one of its lowest six vibrational states, and focusing on the vibrational transitions to the lowest ten vibrational levels, when energetically accessible. Table I shows the energies of these latter levels relative to $v_{i}^{+}=0$ of the target.

The calculations have been performed by taking into account both the direct and the indirect mechanisms, the reaction matrix being evaluated in the second order, and all their vibrational levels - 81, 66 and 50 respectively, associated to open or closed ionization channels, according to the total energy of the system - have been fully accounted.

The cross sections have been calculated for all the relevant symmetries listed in figs. 1 and 2, for collision energies of the incident electron ranging between $10^{-5}$ and $2.3 \mathrm{eV}$, with an energy step of $0.01 \mathrm{meV}$. These cross sections have been summed up to obtain the global cross sections.

The global DR, EC, VE and VdE cross sections for target cations having initial vibrational levels $v_{i}^{+}=0,1$ and 5 are shown in figure 3 (a), (b) and (c) panels respectively. The vertical dark-green dashed lines in the mid and upper panels of figure 3 mark the energy below which the calculations are the most accurate. Above these thresholds the calculations neglect the role of the higher lying dissociative states of the neutral.

Nevertheless, the data displayed continue to be reason- 


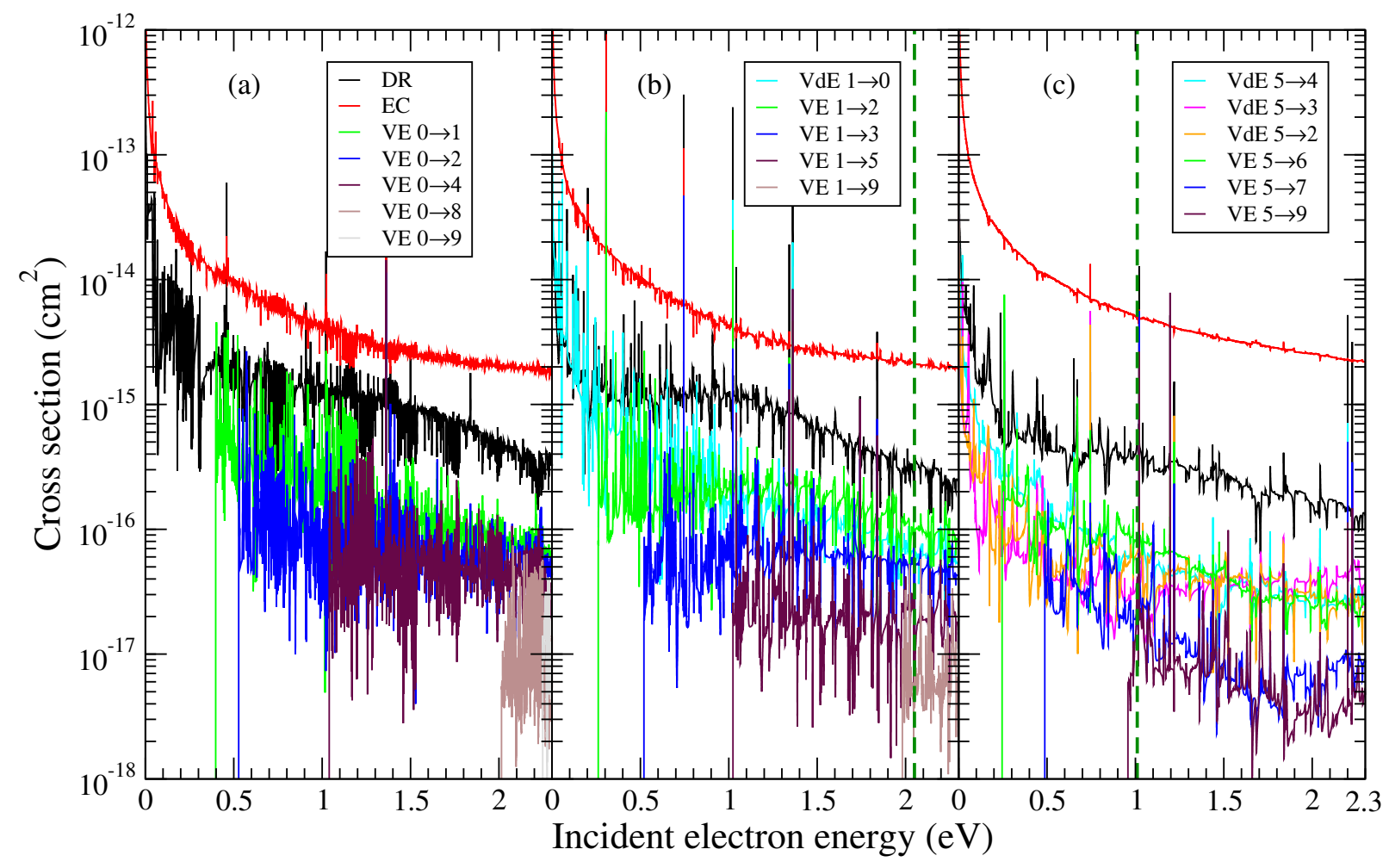

FIG. 3. Global DR, EC, VE and VdE cross sections of the $\mathrm{N}_{2}^{+} v_{i}^{+}=0$ (a), $v_{i}^{+}=1$ (b) and $v_{i}^{+}=5$ (c) as a function of the collision energy. For vibrational transitions (VE and VdE) we label the processes as transitions from the initial to the final vibrational leveles of the target. The vertical dashed dark-green line gives the precision limit of the calculations (for details see text).

ably correct above these thresholds because these dissociative states penetrate into the ionization continuum well above these thresholds, forming favourable/non-vanishing FranckCondon overlaps with the target electronic states at even higher collision energies. This Franck-Condon overlap is proportional with the first order term of the direct cross section. In addition the couplings of these dissociative states with the Rydberg series are generally weaker, leading to less important cross sections in second order.

The direct mechanism is responsible for the background $1 / E$ behaviour of the cross sections, while the indirect one through the temporary capture into the Rydberg states produces all the resonance structures dominating the cross sections.

Among all the processes studied here the elastic collision (red curves in fig. 3) predominates, their cross sections being at least one order of magnitude higher than those obtained for the dissociative recombination (black curves). The global DR cross section increases as we change the initial vibrational state of the target by unity and starts to decreases as we arrive at $v_{i}^{+}=5$. While the vibrational de-excitation (cyan curves for initial vibrational levels higher than 0 ) are in competition with the DR cross section, at higher collision energies their overall cross section values are at least with a factor of 5 smaller than those of the DR. The vibrational excitations (green, blue, violet, maroon, etc. curves) show threshold effects at the collision energies where they become open. Moreover, one can see that for a given initial vibrational level $v_{i}^{+}$the $\left|\Delta v^{+}\right|=1$ vibrational transitions are the most probable ones, decreasing monotonically with $\left|\Delta v^{+}\right|$for the transitions between more distant levels.

Figure 4 shows the thermal rate coefficients of all processes for the six lowest initial vibrational levels of $\mathrm{N}_{2}^{+}$. The green dashed line gives the precision limits of our calculation expressed now in electron temperatures.

The EC (dashed violet line in figure 4), DR (solid black line) and VdE (symbols and thick coloured lines) rate coefficients decrease monotonically with the temperature, while the VE (thin coloured lines) ones increase, partly because of the threshold behaviour of their corresponding cross sections. The largest rate coefficients we obtained are those for the resonant EC process, which is followed by the DR. With the exception of the $v_{i}^{+}=1$ case the $\mathrm{VdE}$ rate coefficients are smaller than those for the DR. At $v_{i}^{+}=1$, the DR is in competition with $\mathrm{VdE}$ but, for $v_{i}^{+}>1 \mathrm{DR}$ exceeds $\mathrm{VdE}$ with a factor of $2-5$. We can see from figure 4 that the VE process is relatively important at high electron temperatures only. Moreover, higher we go with the initial vibrational quantum number of the target cation, more probable VE becomes.

And finally, in order to allow the versatile implementation of the rate coefficients in kinetics modelling codes, we have fitted them with Arrhenius-type formulas. The calculated rate coefficients for the dissociative recombination and for the elastic collisions of electrons with $\mathrm{N}_{2}^{+}$on each of its lowest 6 vibrational levels $\left(v_{i}^{+}=0,1, \ldots, 5\right)$ have been interpolated under the mathematical form: 




FIG. 4. Maxwell rate coefficients for all the relevant electron-induced processes on $\mathrm{N}_{2}^{+}$initially on $v_{i}^{+}=0-5$ vibrational levels : Dissociative recombination (black line), elastic collisions (indigo dashed line), vibrational excitation (thin coloured lines) and vibrational de-excitation (symbols and thick coloured lines). For the vibrational excitations all the transitions are shown up to $v_{f}^{+}=9$ with the lowest transition being labeled on each figure. The excitation and the de-excitation up to the final vibrational quantum numbers are given. The green dashed line gives the precision limit of our calculation given in temperatures (for details see text).

TABLE II. List of the fitting parameters used in formula (5), temperature regions and root mean squares for the EC and DR rate coefficients of $\mathrm{N}_{2}^{+}\left(v_{i}^{+}=0-5\right)$.

\begin{tabular}{ccccccc}
\hline & $v_{i}^{+}$ & Temperature range & $\begin{array}{c}A \\
\left(\mathrm{~cm}^{3} \mathrm{~s}^{-1} \mathrm{~K}^{-\alpha}\right)\end{array}$ & $\alpha$ & $\begin{array}{c}B \\
(K)\end{array}$ & $\mathrm{RMS}$ \\
\hline EC & 0 & $100 \leq \mathrm{T} \leq 5000$ & $3.37355 \times 10^{-5}$ & -0.498093 & -1.97069 & 0.0095 \\
& 1 & $100<\mathrm{T} \leq 5000$ & $3.35449 \times 10^{-5}$ & -0.487562 & -2.85638 & 0.0086 \\
& 2 & $100<\mathrm{T} \leq 5000$ & $4.29406 \times 10^{-5}$ & -0.516353 & 1.01360 & 0.0050 \\
& 3 & $100<\mathrm{T} \leq 5000$ & $4.32596 \times 10^{-5}$ & -0.512870 & 3.42679 & 0.0023 \\
& 4 & $100<\mathrm{T} \leq 5000$ & $4.53781 \times 10^{-5}$ & -0.519476 & 1.46782 & 0.0029 \\
& 5 & $100<\mathrm{T} \leq 5000$ & $4.32166 \times 10^{-5}$ & -0.511299 & 2.01044 & 0.0022 \\
\hline $\mathrm{DR}$ & 0 & $100 \leq \mathrm{T} \leq 700$ & $1.56020 \times 10^{-5}$ & -0.679449 & 53.0333 & 0.0051 \\
& & $700<\mathrm{T} \leq 5000$ & $2.27099 \times 10^{-7}$ & -0.129049 & -388.399 & 0.0040 \\
& 1 & $100 \leq \mathrm{T} \leq 900$ & $1.11178 \times 10^{-7}$ & -0.120373 & -41.6843 & 0.0049 \\
& & $900<\mathrm{T} \leq 5000$ & $2.60704 \times 10^{-8}$ & 0.086645 & -85.4307 & 0.0055 \\
& 2 & $100 \leq \mathrm{T} \leq 1000$ & $1.54430 \times 10^{-6}$ & -0.345836 & -28.1712 & 0.0053 \\
& & $1000<\mathrm{T} \leq 5000$ & $7.09592 \times 10^{-6}$ & -0.544674 & 145.630 & 0.0030 \\
& 3 & $100 \leq \mathrm{T} \leq 5000$ & $1.53033 \times 10^{-6}$ & -0.438705 & -19.1368 & 0.0088 \\
& 4 & $100 \leq \mathrm{T} \leq 460$ & $5.25573 \times 10^{-8}$ & 0.042384 & -76.5345 & 0.0032 \\
& & $460<\mathrm{T} \leq 5000$ & $3.42600 \times 10^{-6}$ & -0.539469 & 208.028 & 0.0031 \\
& 5 & $100 \leq \mathrm{T} \leq 5000$ & $7.20627 \times 10^{-7}$ & -0.394354 & -7.90548 & 0.0106 \\
\hline
\end{tabular}

$$
k^{f i t t}(T)=A T^{\alpha} \exp \left[-\frac{B}{T}\right]
$$

over the electron temperature range $100 \mathrm{~K} \leq T_{e} \leq 5000 \mathrm{~K}$ and/or for rate coefficients larger then $10^{-14} \mathrm{~cm}^{3} \mathrm{~s}^{-1}$, as displayed in fig. 4. The $A, \alpha$ and $B$ fitting parameters used in equation (5) together with the temperature ragions are listed in table II for EC and DR, and in table III for VE and VdE processes. The efficiency of the fitting are characterised with the Root Mean Squares, and we were able to reproduce the MQDT rate coefficients with a precision higher than $97 \%$.

\section{v. CONCLUSIONS}

The present work extends considerably our previous study of the dissociative recombination of $\mathrm{N}_{2}^{+}$with electrons ${ }^{22}$. Making use of the molecular data set calculated in Refs. ${ }^{22,34,35}$ and of our step-wise MQDT method, we have performed calculations for the lowest 6 vibrational levels of the target cation in collision with electrons having kinetic energy up $2.3 \mathrm{eV}$ and, in the case of thermal equilibrium, electronic temperature up to $5000 \mathrm{~K}$. We have provided cross sections and rate coefficients for resonant elastic scattering, dissociative recombination, vibrational excitation and de-excitation of $\mathrm{N}_{2}^{+}$molecular cation, important for the detailed kinetic modelling of cold astrophysical, atmospheric and laboratory plasmas.

\section{ACKNOWLEDGMENTS}

The authors acknowledge support from Fédération de Recherche Fusion par Confinement Magnétique (CNRS, CEA and Eurofusion), La Région Normandie, FEDER and LabEx EMC3 via the projects PTOLEMEE, Bioengine, EMoPlaF, COMUE Normandie Université, the Institute for Energy, 
TABLE III. List of the fitting parameters used in formula (5), temperature regions and root mean squares for the VE and VdE rate coefficients of $\mathrm{N}_{2}^{+}\left(v_{i}^{+}=0-5\right.$ and $\left.v_{f}^{+}=9\right)$. The lines having bold $v_{f}^{+}$values belong to $\mathrm{VdE}$.

\begin{tabular}{|c|c|c|c|c|c|c|c|c|c|c|c|c|c|}
\hline$v_{i}$ & $v_{f}$ & $\begin{array}{c}\text { Temperature range } \\
(\mathrm{K})\end{array}$ & $\begin{array}{c}A \\
\left(\mathrm{~cm}^{3} \mathrm{~s}^{-1} K^{-\alpha}\right)\end{array}$ & $\alpha$ & $\begin{array}{c}B \\
(K)\end{array}$ & RMS & $\overline{v_{i}^{+}}$ & $v_{f}^{+}$ & $\begin{array}{c}\text { Temperature range } \\
(\mathrm{K})\end{array}$ & $\begin{array}{c}A \\
\left(\mathrm{~cm}^{3} \mathrm{~s}^{-1} K^{-\alpha}\right)\end{array}$ & $\alpha$ & $\begin{array}{c}B \\
(K)\end{array}$ & K \\
\hline \multirow[t]{13}{*}{0} & 1 & $270 \leq \mathrm{T} \leq 5000$ & $3.35476 \times 10^{-6}$ & -0.584062 & 4525.43 & 0.0073 & 3 & $\mathbf{0}$ & $100 \leq \mathrm{T} \leq 700$ & $3.69265 \times 10^{-7}$ & -0.399263 & -29.4462 & 0.0087 \\
\hline & 2 & $400 \leq \mathrm{T} \leq 1000$ & $9.13678 \times 10^{-7}$ & -0.553899 & 6100.88 & 0.0021 & & & $700<\mathrm{T} \leq 5000$ & $1.88955 \times 10^{-6}$ & -0.594995 & 205.425 & 0.0045 \\
\hline & & $1000<\mathrm{T} \leq 5000$ & $3.04596 \times 10^{-7}$ & -0.420925 & 5907.02 & 0.0028 & & 4 & $100 \leq \mathrm{T} \leq 560$ & $3.67969 \times 10^{-7}$ & -0.546948 & 2807.96 & 0.0290 \\
\hline & 3 & $600 \leq \mathrm{T} \leq 1500$ & $9.67070 \times 10^{-7}$ & -0.509170 & 3228.55 & 0.0062 & & & $560<\mathrm{T} \leq 5000$ & $1.29198 \times 10^{-7}$ & -0.410534 & 2682.71 & 0.0112 \\
\hline & & $1500<\mathrm{T} \leq 5000$ & $4.98432 \times 10^{-6}$ & -0.699380 & 9626.89 & 0.0040 & & 5 & $440 \leq \mathrm{T} \leq 1300$ & $4.82997 \times 10^{-7}$ & -0.335457 & 5887.42 & 0.0106 \\
\hline & 4 & $850 \leq \mathrm{T} \leq 2000$ & $9.30628 \times 10^{-8}$ & -0.292379 & 11989.3 & 0.0054 & & & $1300<\mathrm{T} \leq 5000$ & $8.49983 \times 10^{-9}$ & -0.137448 & 5427.34 & 0.0084 \\
\hline & & $2000<\mathrm{T} \leq 5000$ & $8.81469 \times 10^{-7}$ & -0.546342 & 12651.0 & 0.0033 & & 6 & $650 \leq \mathrm{T} \leq 1700$ & $1.54145 \times 10^{-7}$ & -0.532191 & 9039.15 & 0.0032 \\
\hline & 5 & $1000 \leq \mathrm{T} \leq 5000$ & $8.92493 \times 10^{-7}$ & -0.596125 & 14871.8 & 0.0066 & & & $1300<\mathrm{T} \leq 5000$ & $9.06525 \times 10^{-10}$ & 0.052019 & 7617.98 & 0.0111 \\
\hline & 6 & $1200 \leq \mathrm{T} \leq 2500$ & $1.44702 \times 10^{-6}$ & -0.667566 & 17980.5 & 0.0022 & & 7 & $850 \leq \mathrm{T} \leq 2000$ & $2.49601 \times 10^{-8}$ & -0.300658 & 11333.8 & 0.0091 \\
\hline & & $2500<\mathrm{T} \leq 5000$ & $5.49126 \times 10^{-6}$ & -0.815886 & 18422.2 & 0.0009 & & & $2000<\mathrm{T} \leq 5000$ & $4.15424 \times 10^{-9}$ & -0.093853 & 10888.7 & 0.0003 \\
\hline & 7 & $1500 \leq \mathrm{T} \leq 5000$ & $1.65552 \times 10^{-6}$ & -0.696318 & 21345.0 & 0.0190 & & 8 & $1100 \leq \mathrm{T} \leq 5000$ & $2.27558 \times 10^{-9}$ & -0.079760 & 13723.8 & 0.0189 \\
\hline & 8 & $1800 \leq \mathrm{T} \leq 5000$ & $9.86709 \times 10^{-8}$ & -0.470871 & 23504.3 & 0.0071 & & 9 & $1300 \leq \mathrm{T} \leq 5000$ & $3.06856 \times 10^{-7}$ & -0.645208 & 17278.8 & 0.0034 \\
\hline & 9 & $2000 \leq \mathrm{T} \leq 5000$ & $1.34002 \times 10^{-6}$ & -0.780327 & 26309.7 & 0.0025 & 4 & 3 & $100 \leq \mathrm{T}$ & $6.37863 \times 10^{-8}$ & $\begin{array}{l}-0.1 \\
\quad\end{array}$ & -28.4720 & 0.0082 \\
\hline \multirow[t]{13}{*}{1} & $\mathbf{0}$ & $100 \leq \mathrm{T} \leq 5000$ & $1.45341 \times 10^{-6}$ & $-0.4^{\prime}$ & 19.3876 & 0.0195 & & & & $6.36181 \times 10^{-6}$ & -0.8 & 192.216 & 0.0072 \\
\hline & 2 & & 200912 & & 3146.96 & 0.0154 & & 2 & 1 & $6.55402 \times 10^{-8}$ & & & 0.0227 \\
\hline & & & -8 & & 2326.31 & 0.0076 & & & & & & & 0.014 \\
\hline & 3 & & 70 & & 6137.15 & 0.0011 & & 1 & & $2.32972 \times 10^{-8}$ & & & 0.0057 \\
\hline & & & 8 & & 5736.89 & 0.0040 & & & & 2.2 & & & 0.0044 \\
\hline & 4 & & 552686 & & 8726.55 & 0.0040 & & $\mathbf{0}$ & & $2.12911 \times 10^{-9}$ & & & 0.0183 \\
\hline & & & & & 9262.99 & 0.0065 & & & & $2.10016 \times 1$ & & & 0.0066 \\
\hline & 5 & & & & 12008.4 & 0.0031 & & 5 & & 7.6 & & & 0.0217 \\
\hline & 6 & & & & 1 & 0.0 & & & & $2.43298 \times 1$ & & & 0.0 \\
\hline & & & & & & 0. & & 6 & & & & & \\
\hline & 7 & & & & 167 & 0.0 & & & & & & & \\
\hline & 8 & & & & 20348.3 & 0.0062 & & 7 & & & & & 0.0036 \\
\hline & 9 & & & -0.3 & 22692.0 & 0.0027 & & & & 7.3 & -0 . & 805 & 0.0 \\
\hline \multirow[t]{14}{*}{2} & 1 & & -7 & & -9.07849 & 0.0073 & & 8 & & 4.1 & & 4.1 & 0.0 \\
\hline & & & & & -377.074 & 0.0027 & & & & 3. & & 7.3 & 0.002 \\
\hline & $\mathbf{0}$ & 100 & $662+3$ & -0.4 & 38.4238 & 0.0193 & & 9 & 100 & 1.1 & & 7.4 & 0.0126 \\
\hline & 3 & & -7 & -0.4 & 2977.42 & 0.0005 & & & $2500<\mathrm{T} \leq 5000$ & $1.25100 \times 10^{-8}$ & -0 & 13039.9 & 0.0002 \\
\hline & & & & -0.3 & 2843.67 & 0.0089 & 5 & 4 & & $4.50970 \times 10^{-7}$ & & & 0.0125 \\
\hline & 4 & 400 & $3 \times 10^{-6}$ & -0.800392 & 6144.90 & 0.0036 & & 3 & 10 & 5.8 & & 59 & 0.0073 \\
\hline & & 130 & & -0.268995 & 5056.36 & 0.0148 & & & & -7 & & & 0.0132 \\
\hline & 5 & & $0^{-8}$ & -0.367691 & 8687.59 & 0.0076 & & 2 & $100 \leq \mathrm{T}$ & $6.21686 \times 10^{-8}$ & & & 0.0101 \\
\hline & & 150 & & -0.117668 & 8217.86 & 0.0022 & & $\mathbf{1}$ & 1 & -8 & & 355 & 0.0022 \\
\hline & 6 & & $0^{-8}$ & -0.401678 & 11429.0 & 0.0164 & & & 900 & $1.08934 \times 10^{-6}$ & -0 . & 198.309 & 0.0031 \\
\hline & 7 & 10 & $9.38561 \times 10^{-7}$ & -0.733592 & 14527.8 & 0.0013 & & & 10 & $3.73256 \times 1$ & -0 . & 2.80363 & 0.0122 \\
\hline & & & & -0.5 & 13960.8 & 0.0012 & & & 00 & & & 278 & 0.0 \\
\hline & 8 & & $24 \times 10^{-6}$ & 21 & 17518.6 & 0.0117 & & 7 & 0 & $77 \times 10^{-8}$ & & 5543.78 & 0.0 \\
\hline & 9 & & -7 & 370 & 19732.3 & 0. & & & 00 & & & .63 & 0.0 \\
\hline \multirow[t]{3}{*}{3} & 2 & $\mathrm{~T} \leq 5000$ & $3.60161 \times 10^{-7}$ & -0.490909 & 23.3038 & 0.0108 & & & 600 & 4.1072 & & 14 & 3 \\
\hline & 1 & & $2.52673 \times$ & 0.215750 & -58.7472 & 0.0 & & 9 & $850 \leq \mathrm{T} \leq$ & $7.61666 \times 10^{-7}$ & -0.7 & 11307.6 & 0.0038 \\
\hline & & $700 \leq \mathrm{T}<5000$ & $7.86265 \times 10^{-8}$ & -0.292543 & 41.8077 & 0.0060 & & & $2000<\mathrm{T} \leq 5000$ & $7.87895 \times 10^{-8}$ & -0.489941 & 10618.0 & 0.0036 \\
\hline
\end{tabular}

Propulsion and Environment (FR-IEPE), the European Union via COST (European Cooperation in Science and Technology) action MD-GAS (CA18212), and ERASMUS-plus conventions between Université Le Havre Normandie and University College London. We are indebted to Agence Nationale de la Recherche (ANR) via the project MONA, Centre National de la Recherche Scientifique via the GdR TheMS and the DYMCOM project, and the Institute Pascal, University Paris-Saclay for the warm hospitality during the DYMCOM workshop. This work was supported by the Programme National 'Physique et Chimie du Milieu Interstellaire' (PCMI) of CNRS/INSU with INC/INP co-funded by CEA and CNES. JZsM thanks the financial support of the National Research, Development and Innovation Fund of Hungary, under the K 18 funding scheme with project no. K128621.

\section{REFERENCES}

${ }^{1}$ V. A. Krasnopolsky, "Chemical composition of titan's atmosphere and ionosphere: Observations and the photochemical model," Icarus 236, 83-91 (2014).

${ }^{2}$ J. L. Elliot, D. F. Strobel, X. Zhu, J. A. Stansberry, L. H. Wasserman, and O. G. Franz, "The thermal structure of triton's middle atmosphere," Icarus 143, 425-428 (2020).

${ }^{3}$ V. A. Krasnopolsky, “A photochemical model of pluto's atmosphere and ionosphere,” Icarus 335, 113374 (2020).

${ }^{4}$ L. A. Young, F. Braga-Ribas, and R. E. Johnson, "Volatile evolution and atmospheres of trans-neptunian objects," in The Trans-Neptunian solar system, edited by D. Prialnik, M. A. Barucci, and L. A. Young (Elsevier, The Netherlands, 2020) Chap. 6, pp. 127-151.

${ }^{5}$ R. A. Armstrong, D. M. Suszcynsky, W. A. Lyons, and T. E. Nelson, "Multi-color photometric measurements of ionization and energies in sprites," Geophys. Res. Lett. 27, 653-656 (2000). 
${ }^{6}$ M. R. Torr, "Neutral and ioan chemistry and solar fluxes," J. Geomag. Geoelectr. 35, 131-153 (1983).

${ }^{7}$ H. Lammer, W. Stumptner, G. Molina-Cuberos, S. Bauer, and T. Owen, "Nitrogen isotope fractionation and its consequence for titan's atmospheric evolution,” Planetary and Space Science 48, 529-543 (2000).

${ }^{8}$ Y. L. Yung and J. R. Lyons, "Triton: Topside ionosphere and nitrogen escape,” Geophysical Research Letters 17, 1717-1720 (1990).

${ }^{9}$ J. Annaloro and A. Bultel, "Vibrational and electronic collisional-radiative model in air for earth entry problems," Phys. Plasmas 21, 123512 (2020).

${ }^{10}$ Y. Plastinin, G. Karabadzhak, B. Khmelinin, B. Zemliansky, A. Gorshkov, and G. Zalogin, "Measurements of the uv radiation generated by the soyuz spacecraft transport capsule during reentry," in 45th AIAA Aerospace Sciences Meeting and Exhibit, https://arc.aiaa.org/doi/pdf/10.2514/6.2007815 .

${ }^{11}$ T. Sakakura, N. Murakami, Y. Takatsuji, M. Morimoto, and T. Haruyama, "Contribution of discharge excited atomic $\mathrm{n}, \mathrm{n}_{2}^{*}$, and $\mathrm{n}_{2}^{+}$to a plasma/liquid interfacial reaction as suggested by quantitative analysis," Chem. Phys. Chem. 20, 1467-1474 (2019).

${ }^{12}$ I. A. Morozov, A. S. Mamaev, I. V. Osorgina, L. M. Lemkina, V. P. Korobov, A. Y. Belyaev, S. E. Porozova, and M. G. Sherban, "Structuralmechanical and antibacterial properties of a soft elastic polyurethane surface after plasma immersion $\mathrm{n}_{2}^{+}$implantation," Material Sci. Eng. C 62, 242-248 (2016).

${ }^{13}$ M. K. Sharma and B. K. Saikia, "Discharge conditions and emission spectroscopy of $\mathrm{n}_{2}$ and $\mathrm{n}_{2}^{+}$active species in a variable power dc pulsed plasma used for steel nitriding," Indian J. Pure and Appl. Phys. 46, 463-470 (2008).

${ }^{14}$ J. D. Holcomb and A. Schucker, "Helium plasma skin regeneration: evaluation of skin tissue effects in a porcine model and comparison to nitrogen plasma skin regeneration," Lasers in Surgery and Medicine 52, 23-32 (2020).

${ }^{15}$ E. C. Zipf, "The dissociative recombination of vibrationally excited $\mathrm{n}_{2}^{+}$ions," Geophysical Research Letters 7, 645-648 (1980).

${ }^{16}$ A. J. Cunningham and R. M. Hobson, "Dissociative recombination at elevated temperatures. IV. $\mathrm{n}_{2}^{+}$dominated afterglows," J. Phys. B: Atomic and Molecular Physics 5, 2328-2331 (1972).

${ }^{17}$ S. K. Mitra, “Active nitrogen,” Phys. Rev. 90, 516-521 (1953).

${ }^{18}$ J. Kaplan, “Active nitrogen,” Phys. Rev 73, 494-496 (1948).

${ }^{19}$ M. A. Biondi and S. C. Brown, "Measurement of electron-ion recombination," Phys. Rev 76, 1697-1700 (1949).

${ }^{20}$ C. Noren, F. B. Yousif, and J. B. A. Mitchell, "Dissociative recombination and excitation of $\mathrm{n}_{2}^{+}$, , J. Chem. Soc., Faraday Transactions 2 85, 1697 (1989).

${ }^{21}$ J. R. Peterson, A. L. Padellec, H. Danared, G. H. Dunn, M. Larsson, A. Larson, R. Peverall, C. Strömholm, S. Rosén, M. af Ugglas, and W. J. van der Zande, "Dissociative recombination and excitation of $\mathrm{n}_{2}^{+}$: Cross sections and product branching ratios," J. Chem. Phys 108, 1978-1988 (1998).

${ }^{22}$ D. A. Little, K. Chakrabarti, J. Z. Mezei, I. F. Schneider, and J. Tennyson, "Dissociative recombination of $n+2$ : An ab initio study," Phys. Rev. A 90,
052705 (2014).

${ }^{23}$ S. L. Guberman, "Spectroscopy above the ionization threshold: Dissociative recombination of the ground vibrational level of $n_{2}^{+}$," J. Chem. Phys 137, 074309 (2012).

${ }^{24}$ S. L. Guberman, "The vibrational dependence of dissociative recombination: Cross sections for $n_{2}^{+}$," J. Chem. Phys 139, 124318 (2013).

${ }^{25}$ S. L. Guberman, "The vibrational dependence of dissociative recombination: Rate constants for $n_{2}^{+}$," J. Chem. Phys 141, 204307 (2014).

${ }^{26}$ K. Chakrabarti, D. R. Backodissa-Kiminou, N. Pop, J. Z. Mezei, O. Motapon, F. Lique, O. Dulieu, A. Wolf, and I. F. Schneider, "Dissociative recombination of electrons with diatomic molecular cations above dissociation threshold: Application to h2+ and hd+," Phys. Rev. A 87, 022702 (2013).

${ }^{27}$ O. Motapon, N. Pop, F. Argoubi, J. Z. Mezei, M. D. Epee Epee, A. Faure, M. Telmini, J. Tennyson, and I. F. Schneider, "Rotational transitions induced by collisions of $\mathrm{hd}^{+}$ions with low-energy electrons," Phys. Rev. A 90, 012706 (2014).

${ }^{28}$ M. D. Epée Epée, J. Z. Mezei, O. Motapon, N. Pop, and I. F. Schneider, "Reactive collisions of very low-energy electrons with $\mathrm{h}_{2}^{+}$: rotational transitions and dissociative recombination," Monthly Notices of the Royal Astronomical Society 455, 276-281 (2016).

${ }^{29}$ A. Abdoulanziz, F. Colboc, D. A. Little, Y. Moulane, J. Z. Mezei, E. Roueff, J. Tennyson, I. F. Schneider, and V. Laporta, "Theoretical study of arh+ dissociative recombination and electron-impact vibrational excitation," Monthly Notices of the Royal Astronomical Society 479, 2415-2420 (2018).

${ }^{30}$ J. Z. Mezei, K. Chakrabarti, M. D. Epée Epée, O. Motapon, C. H. Yuen, M. A. Ayouz, N. Douguet, S. F. dos Santos, V. Kokoouline, and I. F. Schneider, "Electron-induced excitation, recombination, and dissociation of molecular ions initiating the formation of complex organic molecules," ACS Earth Space Chem. 3, 2376 (2019).

${ }^{31}$ D. O. Kashinski, D. Talbi, A. P. Hickman, O. E. Di Nallo, F. Colboc, K. Chakrabarti, I. F. Schneider, and J. Z. Mezei, "A theoretical study of the dissociative recombination of $\mathrm{sh}^{+}$with electrons through the ${ }^{2} \pi$ states of sh," J. Chem. Phy. 146, 204109 (2017).

${ }^{32}$ M. J. Seaton, "Quantum defect theory," Reports on Progress in Physics 46, 167 (1983).

${ }^{33} \mathrm{~J}$. Tennyson, "Electron-molecule collision calculations using the $R$-matrix method," Phys. Rep. 491, 29 - 76 (2010).

${ }^{34}$ D. A. Little and J. Tennyson, "An r-matrix study of singlet and triplet continuum states of n 2,' J. Phys. B: At. Mol. Opt. Phys. 47, 105204 (2014).

${ }^{35}$ D. A. Little and J. Tennyson, "An r-matrix study of singlet and triplet continuum states of n 2,” J. Phys. B: At. Mol. Opt. Phys. 47, 105204 (2014).

${ }^{36}$ H.-J. Werner, P. J. Knowles, G. Knizia, F. R. Manby, and M. Schütz, "Molpro: a general-purpose quantum chemistry program package," WIREs Comput Mol Sci 2, 242-253 (2012). 